\title{
Lapurdum
}

Euskal ikerketen aldizkaria | Revue d'études basques |

Revista de estudios vascos | Basque studies review

$18 \mid 2014$

Numéro XVIII

\section{¿Cómo estudiar el cambio de código ? Incorporación de diferentes metodologías en el caso de varias comunidades bilingües}

\section{Amaia Munarriz y Maria del Carmen Parafita Couto}

\section{CpenEdition}

Journals

Edición electrónica

URL: http://journals.openedition.org/lapurdum/2494

DOI: 10.4000/lapurdum.2494

ISSN: 1965-0655

Editor

IKER

Edición impresa

Fecha de publicación: 1 octubre 2014

Paginación: 43-73

ISBN: 978-2-86781-409-X

ISSN: $1273-3830$

Referencia electrónica

Amaia Munarriz y Maria del Carmen Parafita Couto, « ¿ Cómo estudiar el cambio de código ?

Incorporación de diferentes metodologías en el caso de varias comunidades bilingües », Lapurdum [En línea], 18 | 2014, Publicado el 25 mayo 2016, consultado el 30 abril 2019. URL : http:// journals.openedition.org/lapurdum/2494; DOI : 10.4000/lapurdum.2494 


\title{
¿Cómo estudiar el cambio de código? Incorporación de diferentes metodologías en el caso de varias comunidades bilingües ${ }^{1}$
}

\author{
Amaia MUNARRIZ \\ Universidad del País Vasco UPV/EHU \\ M. Carmen PARAFITA COUTO \\ Leiden University
}

\begin{abstract}
Resumen
El estudio del cambio de código y más específicamente de las restricciones que rigen el habla bilingüe es uno de los temas en pleno auge en la lingüística. Se han propuesto varios modelos teóricos que intentan dar cuenta de los fenómenos que suceden principalmente en "puntos de conflicto", es decir, en estructuras en las que las gramáticas de las lenguas difieren. El objetivo de este trabajo es ofrecer una visión de conjunto de las diferentes metodologías para estudiar las características gramaticales de habla bilingüe en los puntos de conflicto, en base a los resultados obtenidos en estudios ya existentes de algunas comunidades bilingües. Se ofrecerá una breve descripción de algunas de las metodologías usadas, desde métodos más naturales (datos de corpus) hasta métodos más experimentales como las tareas de elicitación (semi-espontánea) y los juicios de aceptabilidad, así como algunos más recientes basados en la medición de la respuesta electrofisiológica mediante potenciales evocados. Se analizará en qué medida permiten los datos obtenidos mediante los métodos expuestos elucidar la teoría lingüística, mediante los resultados obtenidos en estudios recientes de bilingües galés-inglés, euskera-castellano, y papiamento-holandés. Se presentarán los resultados más relevantes de
\end{abstract}

1. Este trabajo surgió a raíz de una comunicación presentada en el curso de verano "Bilingüismo y contacto lingüístico en el País Vasco y en otras comunidades / Elebitasuna eta hizkuntza kontaktua Euskal Herrian eta beste Erkidego batzuetan" celebrado en 2013 en Donostia/San Sebastián. Desde aquí queremos agradecer a los organizadores del curso Beñat Oyharçabal e Irantzu Epelde, por la invitación a participar en él, así como a la audiencia del curso de verano. Queremos agradecer también a Michael Child y Maria José Ezeizabarrena por sus comentarios y sugerencias. También agradecemos a la Universidad del País Vasco (UFI11/06), al Ministerio de Economía y Competitividad (FFI2012-37884-C03-02), al Gobierno Vasco (IT-676-13), al British Academy (Small Research Grant) y a las universidades de Strathclyde (Glasgow) y de Leiden (Research Development Fund) por su apoyo. Este trabajo no hubiera sido posible sin la colaboración desinteresada de los participantes de los estudios de las tres comunidades estudiadas, así como de los colaboradores en dichos estudios. 
dichos estudios con el objetivo de explorar algunos de los conflictos gramaticales que surgen a nivel del sintagma nominal cuando las propiedades de las lenguas que se combinan en el cambio de código difieren, tal es el caso de a) el orden del adjetivo con respecto al del sustantivo (galés-inglés y papiamento-holandés), y b) la existencia o no de género gramatical (euskera-castellano). En general, los estudios corroboran la necesidad de integrar distintas metodologías para poder analizar en profundidad tanto las propiedades lingüísticas como las extralingüísticas del cambio de código.

\section{Abstract}

The study of code-switching and more specifically the restrictions that govern bilingual speech have recently received a lot of attention in linguistics research. Several theoretical models have been proposed mainly to account for what happens at "conflict sites", that is, where the grammars of the two languages differ. The aim of the present study is to provide a general overview of the different methodologies available to evaluate which factors determine the resolution of grammatical conflict in bilingual speech. Reference will be made to the results that previous studies obtained in some bilingual communities. Some of the data gathering methods will be described, from more naturalistic methods (corpus data) to those which are more experimental in nature, such as (semi-spontaneous) elicitation behavioral tasks and auditory acceptability judgment tasks or more novel methods such as the electrophysiological responses measured by means of event related potentials. We will discuss how the data obtained through different methods may cast light on theoretical linguistics based on recent studies on Welsh-English, Basque-Spanish and Papiamento-Dutch bilinguals. The main results of these studies will be presented in order to explore some of the grammatical conflicts that arise in the nominal phrase when the properties of the languages differ. In particular, we will focus on a) the relative order of the noun and the adjective (Welsh-English and Papiamento-Dutch), and b) the presence or absence of grammatical gender (Basque-Spanish). In general, the results corroborate the need for integrating various methodologies in order to analyze in depth both the linguistic and extralinguistic features of code-switching.

Palabras clave: metodología, habla bilingüe, puntos de conflicto, galés-inglés, euskeracastellano, papiamento-holandés.

Keywords: methods, bilingual speech, conflict sites, Welsh-English, Basque-Spanish, Papiamento-Dutch. 


\section{Introducción}

En muchas comunidades los hablantes usan varias lenguas en la misma conversación e incluso en la misma oración, fenómeno que se conoce como cambio de código (ver Deuchar 2012). Los cambios de código que ocurren en situaciones discursivas naturales indican que las dos lenguas de los bilingües están activas en cierta medida y que los bilingües son capaces de usar de una manera dinámica una sola lengua en determinados momentos y las dos en otros, lo que refleja la flexibilidad del procesamiento del lenguaje, por un lado, y el alto control cognitivo de los hablantes por otro (Van Hell, Litcofsky \& Ting en prensa, 2015).

El estudio lingüístico del cambio de código ha demostrado que los bilingües no cambian entre las lenguas al azar: no se limitan a mezclar las lenguas de manera impredecible y aleatoria sino que parecen seguir patrones y normas específicas. En este sentido, el estudio del cambio de código resulta una de las fuentes de evidencia para desarrollar a) modelos psicolingüísticos de procesamiento en bilingües (de Bot 1992; 2003; Kroll \& Tokowicz 2005) y b) modelos lingüísticos sobre la competencia de los hablantes bilingües (la lengua-I, I language en el marco generativo) según González Vilbazo, Bartlett, Downey, Ebert, Heil, Hoot, Koronkiewicz y Ramos (2013) y MacSwan y McAlister (2010). Además, los datos de cambio de código entre lenguas que difieren en torno a determinadas características/rasgos de estudio pueden ofrecer un argumento empírico para poder elucidar cuestiones lingüísticas (o aportar matices sobre fenómenos lingüísticos) a las que no se puede llegar a través de los datos monolingües (González-Vilbazo et al. 2013).

El estudio de los patrones lingüísticos del cambio de código se remonta a finales de 1970, momento en que divergieron dos líneas de investigación en torno al cambio de código, una dirigida hace las motivaciones sociales y el uso del cambio de código en comunidades bilingües y otra hacia los aspectos gramaticales del cambio de código (MacSwan \& MacAlister 2010). En un trabajo pionero sobre el cambio de código, Poplack (1980) sugirió que los bilingües pueden combinar sus lenguas en la misma oración sólo si la yuxtaposición de elementos de las dos lenguas no viola ninguna regla sintáctica de las dos lenguas, condición que se conoce como la restricción de equivalencia (equivalence constraint). A pesar de que esta propuesta se cuestionó sobre bases empíricas en la misma década (Bentahila \& Davies 1983; Berk-Seligson 1986), ha seguido siendo muy influyente debido a que proporciona una solución de sentido común al problema de cómo los bilingües producen los llamados "puntos de conflicto", es decir, aquellas estructuras en las que las gramáticas de las lenguas difieren. El estudio del cambio de código y más específicamente de las restricciones que rigen el habla bilingüe sigue siendo uno de los temas en pleno auge en la lingüística, ya que aunque se han propuesto varios modelos teóricos (MacSwan 1999; Myers-Scotton 2002) para dar cuenta de los fenómenos que suceden principalmente en "puntos de conflicto", ninguno de los enfoques hasta ahora puede proporcionar una explicación completa de los datos.

La falta de evidencia convergente a favor de los modelos teóricos propuestos puede deberse al hecho de que se han buscado soluciones basadas en un único tipo de datos en lugar de utilizar datos de una gama más amplia de fuentes que permitan abordar el fenómeno desde una perspectiva múltiple. Según Gullberg, Indefrey y Muysken (2009), es indispensable 
tener en cuenta que distintos métodos y técnicas permiten responder a distintas cuestiones. Así, se deben emplear distintas técnicas dependiendo de los objetivos de estudio entre los que se pueden mencionar aspectos sociolingüísticos del cambio de código, sus propiedades gramaticales, el procesamiento de dichas estructuras, las bases neuronales que subyacen a dicho fenómeno, etc. Recientemente ha habido algunos intentos de explicar la validez y fiabilidad del uso de distintos métodos para el estudio del cambio de código (González-Vilbazo et al. 2013; Gullberg et al. 2009; MacSwan y MacAlister 2010; Van Hell et al. en prensa, 2015).

Dado este contexto, el objetivo del presente artículo es ofrecer una visión de conjunto de algunas de las metodologías para profundizar en las características gramaticales del cambio de código. Se pretende abordar una de las cuestiones metodológicas fundamentales, es decir, cuál es o cuales son los métodos más adecuados para estudiar los aspectos gramaticales del cambio de código sin comprometer dicho fenómeno (cf. Gullberg et al. 2009). El enfoque empírico serán los resultados obtenidos en los estudios sobre tres comunidades bilingües: galés-inglés, euskera-castellano y papiamento-holandés. Más concretamente, en estos pares de lenguas se han explorado algunos de los "puntos de conflicto" que surgen a nivel del sintagma nominal cuando las propiedades gramaticales de las lenguas que se combinan en el cambio de código difieren, en este caso a) el orden del adjetivo con respecto al del sustantivo (galés-inglés y papiamento-holandés), y b) la existencia o no de género gramatical (euskera-castellano). Se han combinado diferentes metodologías para estudiar las propiedades estructurales del cambio de código en dichas estructuras con el objeto de estudiar si los datos obtenidos presentan evidencia convergente a favor de un único modelo teórico que aporte la mejor explicación de los datos.

La estructura del artículo es la siguiente. En primer lugar en la sección 2 se ofrecerá una descripción de las metodologías principales para el estudio de las propiedades lingüísticas del habla bilingüe y se discutirán las ventajas y desventajas de cada método. En concreto en la sección 2.1 se revisarán las metodologías no-experimentales haciendo especial referencia a los estudios de corpus que recogen datos naturales. En la sección 2.2 se profundizará en tres metodologías experimentales que han resultado válidas para obtener datos sobre construcciones nominales mixtas: las tareas conductuales de elicitación semi-espontánea y juicios de gramaticalidad de estímulos auditivos (sección 2.2.1) así como tareas electrofisiológicas más novedosas como la de los potenciales evocados (sección 2.2.2). En tercer lugar, se presentarán los resultados más relevantes de los estudios realizados sobre las estructuras nominales mixtas en galés-inglés (sección 3.1), euskera-castellano (sección 3.2) y papiamento-holandés (sección 3.3). Se ofrecerá una visión de conjunto de cómo se han implementado los métodos descritos en la sección 2 con el objetivo de obtener evidencia empírica para esclarecer distintas cuestiones lingüísticas en cada uno de estos pares de lenguas. Finalmente, en las conclusiones se reflexionará sobre las diversas metodologías para el estudio de las propiedades gramaticales así como sobre la evidencia que ofrecen para elucidar la teoría lingüística partiendo de los resultados obtenidos en las tres comunidades bilingües. 


\section{Metodologías para el estudio del habla bilingüe}

Las teorías lingüísticas sobre el habla bilingüe se han desarrollado en base a datos empíricos recolectados mediante diversas metodologías. La Figura 1 ilustra de manera esquemática los métodos lingüísticos principales para recolectar datos empíricos. Por un lado, la metodología no cuantitativa o cualitativa ha predominado durante años en la lingüística, y muchas propuestas teóricas se han basado en la introspección. Sin embargo, con el desarrollo de las disciplinas psicolingüística y neurolingüística a finales del siglo XX y el desarrollo de nuevas técnicas, se ha producido un giro hacia metodologías más cuantitativas, que ofrecen datos empíricos más específicos sin dejar de lado en algunos casos las características más reseñables de la introspección (González-Vilbazo et al. 2013). A continuación se ofrecerá una breve descripción de algunas de las metodologías cuantitativas que han permitido abordar el estudio de las características estructurales del cambio de código.

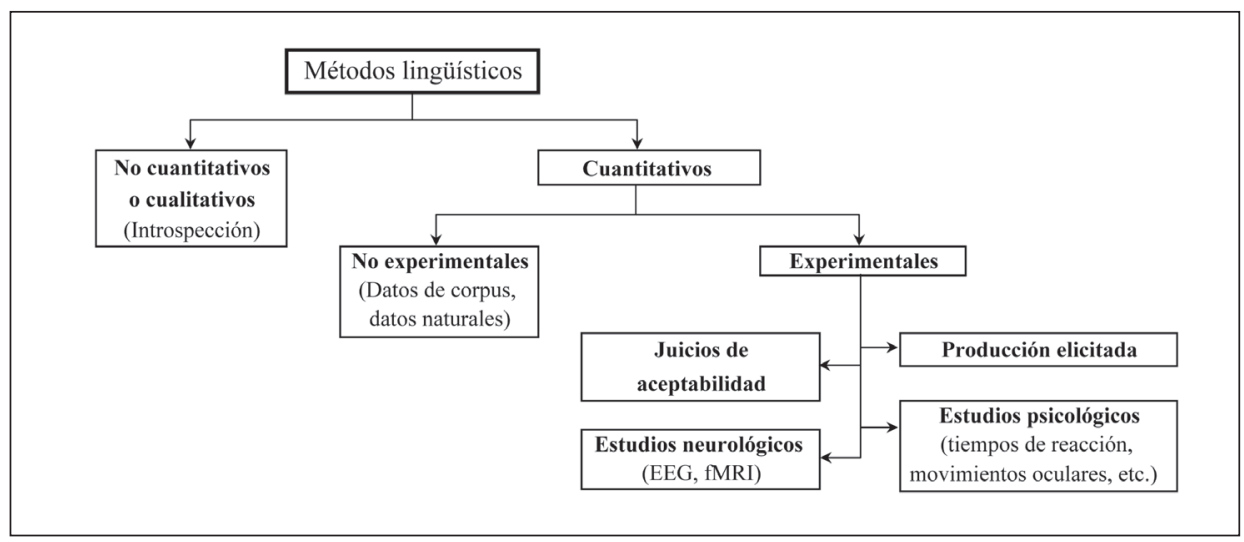

Figura 1. Tipos de métodos utilizados en lingüística para estudios empíricos. (Adaptada de González-Vilbazo et al. 2013: 3).

\subsection{Métodos no experimentales}

Los estudios pioneros sobre el cambio de código se han servido de métodos no experimentales u observacionales en los que se observa el uso lingüístico en diferentes situaciones. Las primeras investigaciones a finales del siglo XIX y comienzos del XX (Weinreich 1953) se basaban en anotaciones escritas de habla natural y en el estudio de textos que contenían ejemplos de cambios de código, aunque dichos métodos no permitían recoger muestras representativas (ver Gullberg, Indefrey \& Muysken, 2009). Gracias al desarrollo de técnicas de grabación, a finales del siglo XX investigadores como Poplack (1980) y Moyer (1992) empezaron a recoger datos naturales mediante grabaciones en varias situaciones comunicativas y así se han construido varios de los corpus sobre habla bilingüe que permiten profundizar en el estudio de diversos aspectos del cambio de código así como de otros fenómenos de interacción. 
El análisis de los corpus de datos naturales ha sido la principal evidencia empírica para abordar las propiedades estructurales y restricciones del cambio de código (Herring, Deuchar, Parafita Couto \& Moro 2010; Jake, Myers-Scotton \& Gross 2002; Poplack 1980). Así, dichos trabajos han dado lugar a teorías muy valiosas sobre las propiedades lingüísticas del fenómeno (Cantone \& MacSwan 2009; Chan 2008; Jake et al. 2002, 2005; MacSwan 2005, 2009; MyersScotton 1992, 2002; Poplack 1980).

La ventaja principal de esta metodología es que permite obtener datos del uso natural del lenguaje sin ningún tipo de manipulación, lo que resulta especialmente relevante cuando el fenómeno de estudio aparece principalmente en habla informal y además está estigmatizado, como es el caso del cambio de código. En concreto, los datos naturales de corpus bilingües han permitido avanzar en el conocimiento de ciertas propiedades estructurales del cambio de código que no se han podido observar en tareas experimentales como por ejemplo los juicios de aceptabilidad, donde se ha observado que ciertas construcciones con cambio de código observadas en habla natural son penalizadas debido a la estigmatización del fenómeno (Parafita, Deuchar \& Fusser 2015). Por otro lado, el análisis cuantitativo de los datos de corpus permite hacer una aproximación de la frecuencia de determinados fenómenos, aunque los resultados pueden variar en gran medida dependiendo de los hablantes, el tema de conversación, etc., lo que dificulta la interpretación de los datos, así como la comparación de diferentes corpus. Según MacSwan y McAlister (2010), el método observacional para recoger datos es el más apropiado para describir y analizar los aspectos sociolingüísticos (motivación social, contexto, etc.) del cambio de código. Buenos ejemplos de ello son los estudios sociolingǘsticos sobre el habla bilingüe euskera-castellano de Lantto (2014) e Ibarra $(2011$, 2013) que se han basado en corpus de datos naturales de jóvenes del gran Bilbao y Navarra, respectivamente.

Sin embargo, los datos naturales no resultan tan efectivos cuando el objeto de estudio son las propiedades gramaticales del cambio de código, debido a las restricciones inherentes de los estudios de corpus. MacSwan y McAlister (2010) mencionan tres limitaciones principales de este método: 1) el problema de la falta de evidencia negativa, 2) el problema de la inducción y 3) el problema de la dificultad para identificar los errores de actuación. Se refieren con el problema de la falta de evidencia negativa a que los datos naturales proporcionan ejemplos de las expresiones posibles (evidencia positiva) pero no de expresiones que no son posibles (evidencia negativa). Sin embargo, es necesario disponer de evidencia de ambos tipos de expresiones (gramaticales y no gramaticales) para que se pueda comprobar si una teoría lingüística sobre el cambio de código resulta empíricamente adecuada (cf. González-Vilbazo et al. 2013). El problema de la inducción hace referencia al hecho de que el que una estructura no se haya encontrado en los datos naturales no significa que dicha estructura no sea posible. Debido a que no hay control sobre los datos, no se garantiza que se obtenga el fenómeno que se desea estudiar de manera espontánea, o en el caso de que se obtengan datos, los datos obtenidos pueden resultar escasos para poder responder preguntas de investigación concretas y determinar la contribución de diferentes factores (MacSwan 2005; Gullberg et al. 2009). En ese sentido, según MacSwan y McAlister (2010) esperar a que se documenten los datos necesarios para poder confirmar o refutar una teoría basada en una estructura específica puede llevar a una frustración continua. Así, no es de extrañar que algunos estudios sobre el cambio de código estén basados en análisis lingüísticos predominantemente descriptivos 
y con un número de ejemplos no muy elevado (Epelde \& Oyharçabal 2010; Ezeizabarrena 2009; Ezeizabarrena \& Munarriz 2012; Oyharçabal, Salaberria \& Epelde 2013, basado en datos del corpus ACOBA de Oyharçabal, Salaberria \& Epelde 2015). A pesar de que el tamaño del corpus puede resultar relevante para el número de ejemplos, cuanto más concreto sea el fenómeno de estudio más difícil resulta obtener un número elevado de ejemplos. Por ejemplo, en el caso del corpus Siarad del galés-inglés (Parafita et al. 2015) se han documentado 160 ejemplos que contienen el adjetivo en una lengua y el sustantivo en la otra en el amplio corpus de 40 horas.

En esta línea, otra de los principales desventajas de esta metodología es el coste que conlleva construir un corpus de datos naturales (Gullberg et al. 2009). A parte del tiempo que conlleva la recolección de datos, hay que añadir el tiempo y las dificultades de la transcripción y codificación. Los investigadores del corpus Siarad (Deuchar, Davies, Herring, Parafita Couto \& Carter 2014) han estimado que han necesitado de una media de noventa minutos para transcribir y codificar un minuto de conversación espontánea.

Finalmente, MacSwan y McAlister (2010) mencionan el problema de la dificultad para identificar los errores de actuación. Según estos autores, los bilingües, al igual que los monolingües, producen errores de actuación en habla natural, y puede suceder que se extraigan conclusiones teóricas basadas en cambios de código que son errores de actuación, por la imposibilidad de identificar dichos errores.

\subsection{Métodos experimentales}

Los métodos experimentales manipulan y restringen la situación que rodea la recolección y ejercen una influencia en los datos que se van a obtener. Entre los métodos experimentales se distinguen dos tipos (Gullberg et al. 2009): a) las consideradas técnicas semi-experimentales, entre las que se encuentran las tareas controladas de elicitación y los juicios de gramaticalidad o aceptabilidad. Por otro lado, se consideran técnicas experimentales las que se realizan en el laboratorio y conllevan diseños estrictos con condiciones experimentales muy específicas. Entre los métodos experimentales, algunos se sirven de técnicas off-line en las que no hay restricción temporal y los participantes pueden reflexionar sobre sus respuestas de una manera bastante libre, y técnicas on-line en las que está en juego el curso temporal del procesamiento lingüístico. Así, las técnicas off-line ofrecen información sobre la respuesta de los participantes, mientras que los métodos on-line permiten obtener información adicional sobre los procesos previos a la respuesta, como por ejemplo el tiempo requerido para procesar las palabras o estructuras, o sus posibles reanálisis. Además, entre los métodos experimentales, algunos permiten obtener datos conductuales, es decir, tipo de respuesta y tiempo requerido para ello, mientras que otros también ofrecen datos neurocognitivos sobre las bases neurológicas del procesamiento.

La ventaja principal de los métodos experimentales es que permiten analizar la actuación lingüística de estructuras concretas (incluso aquellas que resultan menos frecuentes en habla espontánea) de una manera más específica y efectiva. Además, ofrecen la posibilidad de manipular los materiales y así controlar gran parte de las variables que pueden influir en 
los datos (González-Vilbazo et al. 2013). Por el contrario, en comparación con los métodos no-experimentales, se pierde en espontaneidad, y los datos obtenidos mediante algunas de las tareas como los juicios de aceptabilidad pueden ofrecer una visión alejada de la realidad, especialmente en estructuras penalizadas prescriptivamente (Parafita Couto et al. 2015). Asimismo, hay que tener en cuenta que los hablantes pueden desarrollar estrategias concretas a la hora de realizar dichas tareas experimentales, por lo tanto los métodos experimentales requieren de gran meticulosidad durante la preparación de los materiales y el procedimiento a seguir para la recogida de datos.

Los métodos experimentales para analizar las propiedades estructurales del cambio de código más allá del nivel de palabra son escasos/limitados (Gullberg et al. 2009). A continuación se describirán tres métodos experimentales que han permitido analizar propiedades estructurales del cambio de código en puntos de conflicto a nivel intraoracional: dos tareas conductuales de elicitación semi-espontánea (director-matcher task) y juicios de aceptabilidad de estímulos auditivos y una tarea electrofisiológica de potenciales evocados. Para más información sobre los distintos métodos experimentales para al estudio del cambio de código en general, véase Gullberg et al. (2009) y Dussias, Valdes Kroff, Guzzardo Tamargo y Gerfen (2013).

\subsubsection{Tareas conductuales}

\section{Elicitación semi-espontánea}

Las tareas experimentales de elicitación sirven para obtener datos de producción de estructuras lingüísticas concretas. Una de estas tareas que ha resultado útil para el estudio del cambio de código es la conocida como director-matcher task adaptada por Gullberg, Indefrey y Muysken (2009) para elicitar cambios de código en sintagmas nominales (SSNN) complejos (compuestos por determinante, adjetivo y sustantivo) que representan potenciales puntos de conflicto en lenguas en las que el orden del adjetivo difiere (como es el caso de papiamento-holandés). Esta tarea de producción libre que se realiza por parejas es una tarea de comunicación referencial en la que los participantes deben resolver una tarea juntos. Uno de ellos tiene una información necesaria para resolver la tarea y debe comunicarla para que el otro participante pueda servirse de la información y resolver la tarea. La tarea consiste en un juego en el que un participante, el director, debe dar instrucciones al otro, el emparejador, para que haga algo, usando para ello una construcción lingüística determinada. A pesar de que es una tarea libre en la que no se coacciona a los participantes para que produzcan una estructura particular, estimula el cambio de código ya que el experimentador presenta la tarea en modo bilingüe, es decir con cambios de código.

La tarea requiere de dos tableros, un grupo de juguetes/objetos, algunos de los cuales son iguales excepto por el color o alguna otra característica, y un biombo que impide que los participantes vean el tablero ajeno. Los participantes, sentados uno frente al otro y separados por el biombo, disponen de sendos tableros con cuadrículas en las que hay objetos cotidianos. Ambos participantes tienen los mismos objetos pero organizados de una manera diferente. A los participantes se les dice que el objetivo del juego es que terminen con los tableros organizados exactamente de la misma manera tan rápido como puedan y que para ello el director, cuyo rol ha sido asignado previamente a uno de ellos, debe dar instrucciones al otro 
participante sobre cómo distribuir los objetos en el tablero para que al final estén organizados igual que en el suyo. Dichas instrucciones se dan en modo bilingüe con tres objetivos según González-Vilbazo et al. (2013): a) situarlos en un entorno en el que el cambio de código es claramente aceptable y natural b) activar ambas lenguas de los bilingües y c) ejercer un efecto de priming para que los participantes produzcan cambios de código (cf. Gullberg et al. 2009). La tarea se graba para su posterior transcripción.

La ventaja principal de esta tarea es que permite obtener datos semi-espontáneos naturales, gracias a que la atención de los participantes se centra en la tarea organizativa, y se desvía la atención del objetivo de estudio, es decir, las producciones lingüísticas del director. Para evaluar la naturalidad de los datos, es preferible compararlos con los obtenidos en habla espontánea (Gullberg et al. 2009). Además, esta tarea de elicitación resulta más efectiva que los corpus de habla natural para obtener ejemplos de estructuras concretas de una manera que requiere muchísimo menos tiempo (la tarea no suele durar más de cinco minutos). Por ejemplo, en un estudio reciente se obtuvieron 168 ejemplos de cambios de código galés-inglés mediante esta tarea (Parafita Couto et al. 2015), un número significativamente superior al de los 137 ejemplos obtenidos mediante cuarenta horas de conversación espontánea en el corpus Siarad (http://bangortalk.org.uk/). Otra de las ventajas de esta tarea es su adaptabilidad, ya que permite variaciones y modificaciones en función del objetivo de estudio, por lo que resulta apropiada para estudiar varias construcciones lingüísticas, como veremos a continuación. Sin embargo, debido a su carácter libre, la limitación principal de esta tarea es que como ocurre en el habla espontánea, puede que no siempre se consiga que los participantes produzcan las estructuras deseadas (Parafita Couto et al. 2015), por lo que es preferible que se combine con tareas experimentales (cf. Gullberg et al. 2009).

\section{Juicios de aceptabilidad de estímulos auditivos}

Tradicionalmente, los juicios de gramaticalidad o aceptabilidad habituales en estudios lingüísticos son tareas escritas off-line en las que se indaga en el conocimiento gramatical de los hablantes. Este método permite obtener juicios categóricos y escalares de gramaticalidad o aceptabilidad sobre las intuiciones de los hablantes respecto de varias construcciones lingüísticas que se presentan. La tarea de juicios de aceptabilidad de estímulos auditivos es una versión modificada de los juicios de gramaticalidad y permite reducir las actitudes prescriptivas hacia el cambio de código, un fenómeno dominantemente oral (Gullberg et al. 2009). Se ha observado que los estímulos con cambio de código se aceptan en general con más facilidad cuando se presentan de manera auditiva que cuando se presentan en la modalidad escrita (González-Vilbazo et al. 2013). Recientemente, Kornkiewicz y Ebert (2014) han mostrado que en general la modalidad escrita u oral no afecta a la interpretación de los resultados, aunque sí puede ampliar el grado de aceptabilidad o rechazo de ciertos estímulos. Además, la modalidad de presentación auditiva garantiza que todos los participantes escuchen los mismos estímulos. González-Vilbazo et al. proponen esta tarea experimental para estudiar el cambio de código porque combina dos de las ventajas de la introspección: a) ofrece evidencia negativa necesaria para llegar a la lengua-I de los hablantes, y b) su diseño experimental permite un mejor control de los estímulos, así como del procedimiento experimental de la tarea. 
Los lingüistas generativistas tradicionalmente han asumido que los juicios de gramaticalidad están relacionados con el conocimiento gramatical o con la competencia lingüística (Tremblay 2005), mientras que los datos de producción se han tomado como parte de la actuación, incluyendo errores o desviaciones de la competencia. Este punto de vista ha sido objeto de gran controversia (véase Featherston 2007a, b, 2008, 2009; Labov 1975; Schütze 1996). Algunos autores son reticentes al uso de esta metodología debido a la idea de que actitudes prescriptivas pueden interferir en los juicios de gramaticalidad, principalmente en fenómenos que están muy estigmatizados en algunas comunidades como el cambio de código (Parafita Couto et al. 2015; Poplack 1980). En consecuencia, resulta difícil saber si el juicio refleja la competencia del hablante o es consecuencia de la influencia social hacia el cambio de código. A pesar de los intentos por minimizar la influencia no deseable de la prescripción, la principal limitación de esta tarea, es que el grado de aceptabilidad de los enunciados mixtos puede resultar muy bajo como se ha podido observar en algunas comunidades bilingües (galés-inglés, Parafita Couto et al. 2015). Sin embargo, MacSwan y McAlister (2010) no consideran la estigmatización un problema teniendo en cuenta que los lingüistas han realizado investigaciones sin ningún problema con lenguas y variedades de lenguas muy estigmatizadas.

El control de los materiales y la situación experimental resultan especialmente relevantes en esta tarea para que los datos obtenidos sean válidos y fiables, puesto que la elección inadecuada de elementos léxicos puede dar lugar al rechazo de la estructura que lo contiene no porque el cambio no sea gramatical, sino porque no resulta natural con determinado elemento léxico (González-Vilbazo et al. 2013). Estos autores citan como ejemplo un experimento sobre el cambio de código entre taiwanés y español en el que observaron que la inserción de un ítem léxico referente a un arroz típico taiwanés al hacer el cambio de código resultaba en una estructura más natural para los participantes. Por lo tanto resulta muy importante a la hora de diseñar la tarea seleccionar con minuciosidad los elementos léxicos de los estímulos que se van a presentar. Una de las maneras de neutralizar dicho efecto es seleccionar estructuras documentadas en habla espontánea (corpus) y mantener la mayor parte de ítems léxicos constantes, a excepción de los elementos que se quieren estudiar (Parafita Couto et al. 2014; Parafita Couto et al. 2015).

Considerados dichos aspectos metodológicos, en el caso de los estudios que describiremos a continuación, las tareas de juicios de aceptabilidad se realizaron de la siguiente manera. Los participantes se sentaban de manera individual delante de un ordenador que contenía un cajetín de respuestas. Antes de realizar la tarea, se les daban las instrucciones por escrito en las dos lenguas de estudio. En dichas instrucciones se les decía que escucharían varias producciones cortas que contenían fragmentos de habla de cada una de las lenguas y que debían dar su opinión acerca de ellas pulsando una de las respuestas del cajetín (Tabla 1). Con el objetivo de evitar actitudes prescriptivas, durante la consigna se insistía en que no se les preguntaba si las construcciones eran o no correctas, sino si creían que podrían escuchar construcciones de ese tipo en situaciones completamente informales. Antes de comenzar con la tarea, los participantes hacían una pequeña prueba con oraciones que contenían otro tipo de estructuras con cambios de código. 


\begin{tabular}{|l|l|}
\hline Botón & Interpretación \\
\hline$\odot$ & Sí, sí que podría oírlo \\
\hline$\odot$ & No sé, no estoy seguro de si podría oírlo \\
\hline$\odot$ & No, no podría oírlo \\
\hline
\end{tabular}

Tabla 1. Opciones de respuesta para los participantes en la tarea auditiva de juicios de aceptabilidad.

El hecho de presentar las producciones en el ordenador permite además combinar la tarea conductual de juicios con tareas habituales en la psicología experimental como puede ser la medición de los tiempos de respuesta. El uso del programa DMDX para la presentación de los estímulos ha permitido por un lado que los participantes decidiesen el ritmo de aparición de los estímulos y por otro lado medir tanto las respuestas como el tiempo que requerían para responder, lo que puede ofrecer información sobre las respuestas más automáticas e instintivas de los participantes.

\subsubsection{Tareas electrofisiológicas}

Algunos estudios psicolingüísticos recientes se sirven de métodos neurolingüísticos con el objetivo de estudiar los procesos psicológicos que subyacen al cambio de código y su relación con otros procesos cognitivos como el control cognitivo. Uno de estos métodos neurocognitivos es el de los potenciales evocados (en adelante ERPs del inglés event related potentials), una técnica que mide la respuesta eléctrica del cerebro mediante la señal del encefalograma para estudiar/medir los procesos cognitivos relacionados temporalmente con un evento. En el caso de la psicolingüística los potenciales evocados permiten medir la respuesta cerebral (actividad eléctrica neuronal) ante determinados estímulos lingüísticos. La alta resolución temporal de esta técnica la convierte en una valiosa herramienta para el estudio del procesamiento lingüístico, ya que proporciona índices de la actividad cerebral obtenidos de manera simultánea a dicho procesamiento.

Varios estudios han observado que el cerebro emite distintas respuestas, es decir, picos de actividad negativa y positiva con distinta latencia y topografía dependiendo del proceso lingüístico que lleva a cabo. En la literatura neurolingüística se han identificado varios componentes, entre los que resultan de especial relevancia el N400 (negatividad cuyo pico emerge hacia los 400 ms del inicio del estímulo) y el P600 (positividad cuyo pico emerge hacia los 600 ms) que son sensibles a procesos léxico-semánticos y gramaticales, respectivamente. Otro de los componentes asociados a los procesos sintácticos es el LAN (Left Anterior Negativity, negatividad izquierda anterior) que varía en latencia y topografía, dependiendo del tipo de violación lingüística (Gullberg et al. 2009; Kutas, Moreno \& Wicha 2009; Parafita Couto et al. en revisión).

Los potenciales evocados ofrecen respuestas automáticas y medidas precisas para estudiar los efectos del cambio de código en el procesamiento y sirven además para examinar 
los procesos cerebrales que preceden a la producción del cambio de código (Gullberg et al. 2009). Los estudios neurocognitivos en torno al cambio de código se han centrado principalmente en los cambios de lengua a nivel de palabra, tanto de manera aislada como en contexto (ver revisión de Van Hell, et al. en prensa, 2015), con el objeto de ver cómo se procesan esas estructuras en comparación con estímulos sin cambio de lengua. Recientemente se ha desarrollado una nueva línea de estudios cognitivos y neurocognitivos en torno a cambios de código en situaciones más naturales, como frases con significado y cambios de código intraoracionales (Van Hell et al. en prensa, 2015). Sin embargo, dichos estudios están orientados a estudiar la razón por la que suceden los cambios de código, así como los mecanismos que subyacen al cambio de código en los bilingües, como por ejemplo el coste que conlleva.

Parafita Couto et al. (en revisión) han demostrado que esta técnica puede proporcionar evidencia robusta y sin precedentes para evaluar la validez de teorías lingüísticas sobre el cambio de código. En este estudio se pusieron a prueba las predicciones de dos teorías sintácticas con el objeto de analizar de una manera sistemática las predicciones de la teoría lingüística. En concreto, este método permite ver si en determinadas condiciones críticas aparecen componentes de ERPs asociados con violaciones sintácticas.

Según los autores, este método resulta especialmente válido para probar todas las combinaciones posibles de las predicciones teóricas para las que los datos de corpus resultan insuficientes. Además, dicha técnica permite acceder a juicios inconscientes de los hablantes, puesto que se basa en las respuestas cerebrales a un estímulo específico. Por lo tanto se evita la no deseada interferencia de las actitudes prescriptivas que pueden influir en los juicios conscientes que se obtienen en tareas como los juicios de aceptabilidad.

A pesar de que esta técnica experimental parece muy prometedora / alentadora para evaluar teorías lingüísticas, el hecho de que no haya estudios previos que puedan servir como referencia dificulta la interpretación de los datos. Por otro lado, esta técnica experimental requiere de gran minuciosidad a la hora del diseño experimental ya que este condiciona completamente el análisis y la interpretación de los datos, tarea de por sí compleja en las técnicas electrofisiológicas.

En resumen, cada método y técnica permite abordar distintos aspectos sobre el cambio de código y cada uno tiene sus limitaciones. Según Gullberg et al. (2009) hay que abandonar la falsa dicotomía entre métodos "buenos" y "malos" y se debe reconocer y aceptar como fuente de información complementaria la que ofrecen métodos más naturales y ecológicamente válidos y también la de técnicas experimentales más artificiales y controladas. Así, la evidencia convergente de distintas técnicas es la mejor manera de asegurar la validez de los datos. Este enfoque de tareas múltiples permite al investigador analizar si los patrones observados a nivel oracional de manera natural se pueden replicar experimentalmente, objetivo que merece la pena por sí mismo (Gullberg et al. 2009). 


\section{Algunos estudios en comunidades bilingües}

El estudio lingüístico detallado de las propiedades que rigen del cambio de código en los puntos de conflicto requiere igualmente de datos obtenidos mediante la combinación de distintas metodologías. Con el objeto de ver en qué medida permiten los datos obtenidos mediante diferentes metodologías elucidar la teoría lingüística, en esta sección se presentarán algunos estudios realizados en tres comunidades bilingües: galés-inglés, euskera-castellano y papiamento-holandés. Siguiendo el enfoque de tareas múltiples propuesto por Gullberg et al. (2009) se han analizado las características del cambio de código intraoracional a nivel del sintagma nominal en base a datos obtenidos mediante distintas tareas. Más específicamente, se han recolectado datos combinando los métodos presentados en la sección anterior. Las características metodológicas de dichas tareas pasan gradualmente de escenarios interactivos en los que participan más de un participante y en los que los cambios de código pueden ocurrir más fácilmente, a escenarios individuales; de tareas espontáneas y naturalísticas a tareas controladas y experimentales; de tareas donde no hay ninguna restricción para que los participantes produzcan cambios de código a tareas mas controladas (Figura 2).

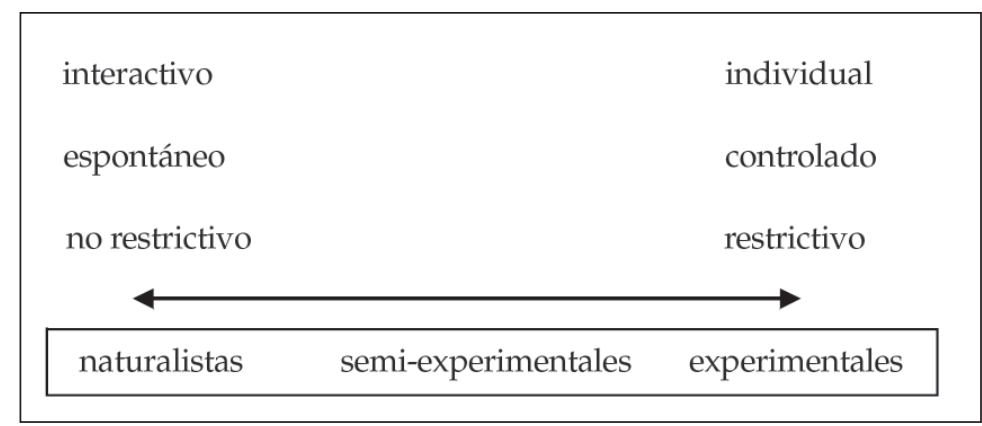

Figura 2. Características de las tareas en el enfoque de tareas múltiples.

\subsection{Galés-inglés}

En galés-inglés, uno de los puntos de conflicto que se ha estudiado es la posición del adjetivo (postnominal en galés y prenominal en inglés) con respeto al sustantivo. Compárese, por ejemplo, red wine "tinto vino" en inglés con gwin coch "vino tinto" en galés. En el habla bilingüe galés-inglés la construcción nominal es entonces un potencial "punto de conflicto". ¿Podemos esperar entonces que un bilingüe produzca por ejemplo, gwin red o red gwin, o wine coch o coch wine? Diferentes teorías hacen predicciones contradictorias sobre las posibilidades que son aceptables. De acuerdo con el modelo del Marco de Lengua Matriz (en adelante MLF del original Matrix Language Framework, Myers-Scotton 2002) el orden de las palabras tiene que ser compatible con la lengua del verbo matriz. Pero para los generativistas Cantone y MacSwan (2009), cuya propuesta se enmarca dentro del Programa Minimalista, es el lenguaje del adjetivo el que determina la posición de la frase nominal en relación con el adjetivo. De esta manera, de acuerdo con el MLF, si la lengua matriz es el galés, se predice que el adjetivo aparezca en posición postnominal, mientras que si la lengua matriz es el inglés, se prevé que el 
adjetivo aparezca en posición prenominal, sin importar que el adjetivo venga del inglés o del galés. En cambio, de acuerdo a las predicciones de Cantone y MacSwan, si el adjetivo viene del inglés aparecerá en posición prenominal y si el adjetivo viene del galés aparecerá en posición postnominal, independientemente de cual sea la lengua matriz de la oración.

Parafita, Deuchar y Fusser (2015) diseñaron un estudio para evaluar los conflictos dentro de las construcciones nominales en el cambio de código galés-inglés utilizando un enfoque de tareas múltiples que comprende (1) datos de corpus de producción espontánea, (2) una tarea de elicitación semi-espontánea, y (3) una tarea de aceptabilidad auditiva.

\subsubsection{Datos de corpus}

Para esta parte del estudio se utilizó un corpus ya existente (el corpus Siarad, ver sección 2.1) de conversaciones informales entre parejas o grupos de personas bilingües galésinglés. A partir de este corpus se extrajeron de forma automática todos los ejemplos de grupos de adjetivo-sustantivo o sustantivo-adjetivo donde el adjetivo y el sustantivo diferían en su lengua. Se encontraron 137 ejemplos, pero todos ellos con el galés como lengua matriz. La tabla 2 muestra los patrones encontrados. Las columnas corresponden a la colocación del adjetivo respecto del sustantivo, las filas indican el orden de las lenguas de los dos elementos.

\begin{tabular}{|l|l|l|l|}
\hline & Sustantivo-Adjetivo & Adjetivo-Sustantivo & Total \\
\hline Galés + Inglés & $36(26,3 \%)$ & $1(0,7 \%)$ & $37(27 \%)$ \\
\hline Inglés + Galés & $93(67,9 \%)$ & $7(5,1 \%)$ & $100(73 \%)$ \\
\hline Total & $129(94,1 \%)$ & $8(5,8 \%)$ & $137(100 \%)$ \\
\hline
\end{tabular}

Tabla 2. Construcciones nominales bilingües en el corpus Siarad. (Adaptada de Parafita Couto et al. 2015).

¿Qué modelo teórico describe los datos adecuadamente? Tomando en consideración que el idioma matriz es galés para todos los ejemplos, el MLF predice una colocación postnominal de los adjetivos. Los resultados para esta posición se muestran en la primera columna de la tabla. De hecho el 94,1\% de los ejemplos se hallan en esta categoría. Por otro lado, la postura generativista de Cantone y MacSwan (2009), predice un predominio de ejemplos correspondientes a una colocación postnominal del adjetivo galés o prenominal del adjetivo inglés (segunda fila). El 73\% de los ejemplos están en esta categoría. Así, parece que el Programa Minimalista tiene un menor grado de acierto en sus predicciones que el MLF a la hora de explicar los resultados del corpus.

Curiosamente, la combinación más frecuente observada en el corpus es la de sustantivo inglés - adjetivo galés $(67,9 \%)$, resultado que es compatible con ambos enfoques.

\subsubsection{Tarea de elicitación semi-espontánea}

Para este experimento se reclutaron 50 participantes bilingües galés-inglés con edades comprendidas entre 18 y 77 años. El 67\% de ellos tiene como primera lengua el galés, el 9\% 
el inglés y el 24\% son bilingües simultáneos galés-inglés. Se adaptó la director-matcher task (ver sección 2.2.1) para estudiar el orden del adjetivo y el sustantivo. En (1) se muestra la lista de los 16 objetos que se utilizaron en la tarea:

(1)

$$
\begin{aligned}
& \text { ratón negro } \\
& \text { ratón blanco } \\
& \text { bolsa de té triangular } \\
& \text { bolsa de té cuadrada } \\
& \text { bolsa de té redonda } \\
& \text { cinta métrica azul } \\
& \text { cinta métrica naranja } \\
& \text { cuerda verde }
\end{aligned}
$$
cadena amarilla
cadena azul
gafas naranjas
gafas azules
calculadora azul
calculadora negra
pulsera naranja
pulsera violeta

Para el análisis de los datos, se extrajeron de las grabaciones las construcciones nominales bilingües que incluyen un adjetivo y un sustantivo ( $\mathrm{y}$ opcionalmente un determinante explícito y / u otros modificadores). Se registraron 168 ejemplos y todos ellos ocurrieron en oraciones que tenían el galés como lengua matriz ${ }^{2}$. Al igual que con los datos del corpus Siarad, no se encontró ningún ejemplo con lengua matriz inglés. A excepción de algunas islas ("embedded language islands") en las que se inserta el adjetivo + sustantivo del inglés como unidad compleja en una oración galesa, los demás adjetivos eran postnominales. Se observaron tres patrones principales (en orden de frecuencia) ${ }^{3}$ : sustantivo inglés + adjetivo galés $(132 / 168,78,6 \%)(2)$, sustantivo galés + adjetivo inglés (15/168, 8,9\%) (3) y sustantivo inglés + adjetivo inglés (13/168, 7,7\%) (4).

(2)

y
Det
a

(3)

$\begin{array}{ll}\text { rhaff } & \text { green } \\ \text { cuerda } & \text { verde } \\ \text { cuerda } & \text { verde }\end{array}$

(4)

$\begin{array}{lll}\text { (4) } & \text { bracelet } & \text { orange } \\ \text { Det } & \text { pulsera } & \text { naranja } \\ \text { La } & \text { pulsera } & \text { naranja }\end{array}$

$\operatorname{glas}^{4}$

azul

azul

calculadora azul

2.- En este estudio se analizó si había correspondencias entre las actitudes de los hablantes hacia el cambio de código y la cantidad de ejemplos de cambios de código. En general, se observó que los participantes que tenían actitudes más negativas produjeron menos construcciones nominales mixtas.

3.- Los 8 ejemplos restantes tenían patrones sintácticos más complejos.

4.- En los ejemplos de este artículo se han indicado mediante cursiva los elementos insertados de la otra lengua. 
Las predicciones generativistas no dan cuenta de los patrones (3) y (4), donde se utiliza un adjetivo inglés en posición postnominal, lo que significa que 28 (17\%) de los 168 ejemplos no corresponde a las predicciones. Sin embargo, todos los ejemplos se pueden explicar mediante el MLF, ya que según este modelo se prevé encontrar adjetivos postnominales en un contexto con lengua matriz galesa.

\subsubsection{Tarea de juicios de aceptabilidad de estímulos auditivos}

Los mismos participantes que tomaron parte en la tarea de elicitación descrita en 3.1.2 completaron también una tarea de juicios de aceptabilidad. La tarea constaba de 84 estímulos bilingües en total, que incluían 36 distractores y ejemplos de práctica. Los 48 estímulos experimentales incluían permutaciones del mismo sintagma nominal en posición de sujeto o de objeto, con el galés o el inglés como lengua matriz, con el determinante en galés o en inglés, con el adjetivo en galés o en inglés, con el sustantivo en galés o en inglés, y además con el adjetivo en posición pre o postnominal. En las oraciones experimentales se mantuvo constante el marco oracional en inglés y solo variaron los elementos del sintagma determinante, para que las diferencias en los juicios solo se debiesen al SN mixto (ver sección 2.2.1).

En general, los estímulos experimentales se consideraron inaceptables, con el promedio más alto de 1,58 en una escala de 1 (inaceptable) a 3 (aceptable) ${ }^{5}$. Sin embargo, los participantes no rechazaron categóricamente los enunciados mixtos, como lo indican las puntuaciones de hasta 2,88 en el caso de alguno de los distractores. Esos distractores que se consideraban más aceptables incluían elementos léxicos frecuentes en el lenguaje cotidiano, mientras que para los estímulos experimentales se eligieron deliberadamente elementos léxicos en inglés que no se oyen normalmente insertados en galés. La elección de elementos léxicos de baja frecuencia se realizó con el objetivo de evitar cualquier posibilidad de que se trataran de préstamos (en vez de cambios de código) del inglés.

Entonces, ¿cómo explican las dos posturas teóricas estos resultados? De acuerdo con el MLF todos los estímulos que tienen galés como lengua matriz y el orden de palabras del galés dentro del sintagma nominal (es decir, un adjetivo postnominal) deben ser más aceptables que aquellos donde hay una falta de correspondencia entre la lengua matriz y el orden de palabras.

La figura 3 muestra los resultados para la lengua matriz inglesa en las columnas de la derecha y para la lengua matriz galesa en las columnas de la izquierda.

5.- En este caso no se observó ninguna correspondencia entre las actitudes de los hablantes hacia el cambio de código y su nivel de aceptación en la tarea de juicios, a diferencia de lo observado en la tarea de producción semi-espontánea. 


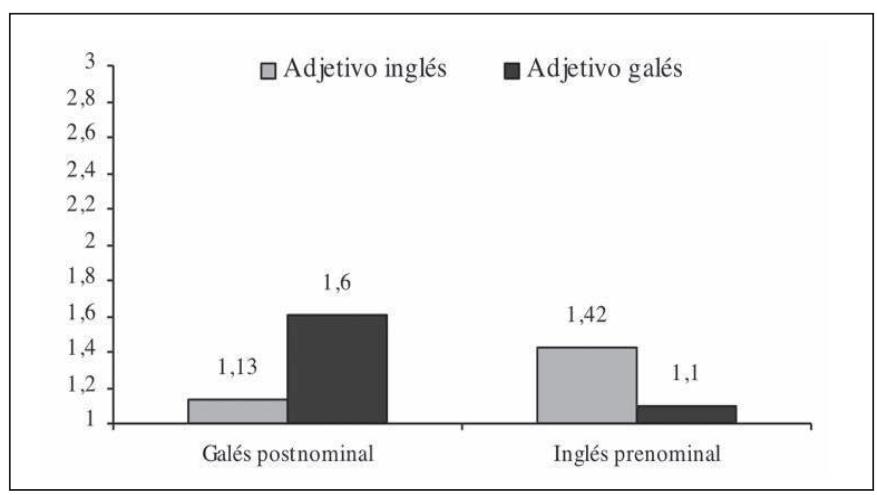

Figura 3. Resultados de lengua matriz y lengua del adjetivo ( $1=$ inaceptable, 3 aceptable). (Adaptada de Parafita Couto et al. 2015).

Los datos de la figura 3 parecen apoyar al MLF, ya que los adjetivos parecen ser más aceptables en posición postnominal cuando el idioma matriz es galés y en posición prenominal cuando el idioma matriz es inglés. Sin embargo, también parece a primera vista que hay apoyo para la predicción generativista de que la lengua del adjetivo determina el orden sustantivoadjetivo. Cuando el adjetivo está en inglés, los más aceptados son los sintagmas con adjetivos prenominales, mientras que cuando el adjetivo es galés, los más aceptados son los sintagmas con adjetivos postnominales. Sin embargo, antes de llegar a la conclusión de que ambos marcos teóricos tienen el mismo nivel de éxito con sus predicciones, debemos notar que este resultado puede reflejar simplemente la preferencia de los bilingües por el uso de sustantivos de la otra lengua en vez de adjetivos.

Los resultados ponen en duda la utilidad de los juicios de gramaticalidad para la investigación del cambio de código y sugieren que se necesita adoptar una gama más amplia de métodos.

\subsubsection{Tarea electrofisiológica}

Siguiendo esta misma línea, Parafita Couto, Boutonnet, Hoshino, Davies, Deuchar y Thierry (en revisión) hacen uso del contraste sintáctico entre el orden del adjetivo y el sustantivo en galés y en inglés para poner a prueba las predicciones de los mismos dos modelos teóricos sobre el cambio de código. Como ya se ha explicado, la posición del adjetivo es estrictamente prenominal en inglés (por ejemplo, blue car 'azul coche') pero estrictamente post-nominal en galés (por ejemplo, car glas 'coche azul'). La aceptabilidad de las construcciones nominales mixtas se investigó mediante una tarea de verificación oracional y potenciales evocados (ERPs). Parafita Couto et al. manipularon (1) la lengua matriz, que se correspondía con la del verbo de la oración, (2) la lengua del adjetivo, (3) la lengua del sustantivo, y (4) el orden de las palabras.

Mientras que el MLF predice que un adjetivo galés en posición prenominal dentro de una oración con lengua matriz inglés sería aceptable, también predice la existencia de una 
violación de los adjetivos en inglés en posición prenominal dentro de una oración con lengua matriz galesa. Sin embargo, las predicciones del programa minimalista (MP), únicamente basadas en la lengua del adjetivo, son exactamente las opuestas.

Parafita Couto et al. han observado un pico negativo en la región frontal del cerebro entre 280-340 ms en la condición que el MLF preveía que induciría una violación. En el mismo estudio hicieron una comparación directa entre respuestas electrofisiológicas provocadas por adjetivos galeses e ingleses en oraciones con cambio de código. Esta comparación no mostró diferencia significativa alguna en la ventana temporal citada.

En resumen, Parafita et al. (2015) encontraron que los datos del corpus y los de la tarea de elicitación eran compatibles, dando apoyo a la superioridad relativa del modelo MLF. Sin embargo, sólo una pequeña proporción de los datos podía distinguir entre los dos modelos. La combinación más frecuente en sintagmas nominales mixtos era la de sustantivo inglés seguido de adjetivo galés. Dado que el galés era siempre la lengua matriz, los datos eran compatibles tanto con las predicciones del MLF como con las predicciones minimalistas. Los datos de la tarea de juicios de aceptabilidad pueden indicar que este tipo de tarea no es muy útil en la investigación del cambio de código ya que todos los estímulos experimentales fueron considerados inaceptables. Esto es probablemente debido a la estigmatización del cambio de código en esta comunidad. Por lo tanto, estos autores recurrieron a la técnica de potenciales evocados (ERPs) y encontraron evidencia a favor del MLF. Este estudio ofrece un primer ejemplo de la forma en que los métodos neurocognitivos pueden elucidar la teoría lingüística, especialmente en los casos en que los datos de producción (corpus y elicitación) y los datos de juicios de gramaticalidad parecen insuficientes para probar todas las combinaciones posibles de las predicciones teóricas.

\subsection{Euskera-castellano}

Otro conflicto gramatical a nivel del sintagma nominal se manifiesta entre el determinante y el sustantivo en lenguas en las que una de ellas tiene género gramatical y la otra no, como es el caso del euskera (no tiene género gramatical en el componente nominal) y el castellano (distingue entre masculino y femenino). La pregunta que surge es qué factores determinarán la asignación de género, es decir, qué género tendrá el determinante cuando aparece junto con un sustantivo (en una lengua) que carece de él. Este conflicto ha sido ampliamente estudiado en inglés (sin género gramatical) y castellano (lengua con género gramatical). Dichos estudios han concluido que a pesar de que el género analógico de los sustantivos (género del sustantivo equivalente en castellano) y en menor medida su terminación fonológica son factores que influyen en la asignación del género, el género masculino es el género por defecto en el habla bilingüe inglés-castellano (Dussias et al. 2013; Jake et al. 2002; Liceras, Fernández-Fuentes, Perales, Pérez-Tattam \& Spradlin 2008), tal y como se ha propuesto para el género en español monolingüe (Harris 1991; Roca 1989, 2005).

En base a los resultados de los estudios sobre SSNN mixtos en inglés-castellano, se puede predecir que el masculino será el género por defecto también en euskera-castellano. Con el objeto de analizar cuáles son los factores que rigen la asignación de género en construcciones 
nominales mixtas con sustantivos en euskera y determinantes en castellano Parafita Couto, Munarriz, Epelde, Deuchar y Oyharçabal (2014) han realizado un estudio con varias tareas que varían en grado de espontaneidad y restricción. Se han recogido datos mediante tres métodos: datos naturales, una tarea semi-experimental de elicitación (director-matcher task) y una tarea auditiva de juicios de gramaticalidad. Los datos naturales han servido como base para estudiar más en detalle el fenómeno de una manera experimental. Además, se han recogido datos sobre la adquisición, capacidad y uso de las lenguas mediante un cuestionario en que también se incluyeron dos preguntas sobre el uso y la actitud hacia el cambio de código castellano-euskera.

\subsubsection{Datos de corpus}

Deuchar, Epelde, Oyharçabal y Parafita Couto (2010) recogieron datos naturales mediante anotaciones escritas entre hablantes nativos de euskera para los que el cambio de código es un fenómeno muy habitual. El corpus se compone de 92 ejemplos de SSNN mixtos con determinante en castellano y sustantivo en euskera. Un análisis cuantitativo de estos datos ha permitido observar que la mayoría de estos ejemplos (72/92, 78,3\%) contiene determinante femenino, puesto que el determinante femenino aparece tanto con sustantivos cuyo género analógico es femenino como con los que tienen equivalente masculino (5). Además, la mayoría de los sustantivos en euskera se insertan con el determinante sufijo $-a$, que crucialmente es homófono a la marca de género femenino en castellano. Tanto el género analógico como la forma fonológica pueden explicar el 53\% (49/92) de los datos, pero en el resto de los ejemplos el factor más relevante resulta ser la terminación fonológica, puesto que explica el 43\% (40/92) de los datos, a diferencia del género analógico que solo explica el 3\% (3/92). En base a esos resultados preliminares, Deuchar et al. (2010) concluyeron que podría haber una reinterpretación del determinante como marca de género femenino.

$$
\begin{aligned}
& \text { ¿Te dan miedo [la-s txakurr-a-s]? } \\
& \text { D.Fem-Pl perro-Det-Pl } \\
& \text { "¿Te dan miedo las perros?" }
\end{aligned}
$$

A fin de poder sustentar este debate con más datos que permitan un análisis más detallado del mismo, se diseñaron dos tareas experimentales una de elicitación y otra de juicios de aceptabilidad.

\subsubsection{Tarea de elicitación semi-espontánea}

Se han analizado datos de 30 hablantes bilingües castellano-euskera cuya primera lengua es castellano y han adquirido euskera en la escuela mediante un sistema de inmersión lingüistica (L1 castellano y L2 temprana euskera), llegando a ser muy competentes en dicha lengua.

La tarea (semi-)experimental de elicitación (director-matcher task, ver sección 2.2.1) se adaptó para el estudio del género, más concretamente con el objetivo de ver si la asignación del género se realiza en base a las características fonológicas del nombre en euskera o al género del sustantivo equivalente en castellano. Se seleccionaron 16 imágenes de objetos y adjetivos 
controlados para género en castellano (8 masculinos y 8 femeninos) y terminación fonológica en euskera ( 8 sustantivos con terminación en - a léxica y 8 con terminación diferente a -a) (Tabla 3).

\begin{tabular}{|c|c|c|c|}
\hline & & \multicolumn{2}{|c|}{ Género en castellano } \\
\hline & & Masculino & Femenino \\
\hline \multirow{4}{*}{$\begin{array}{l}\text { Terminación } \\
\text { fonológica en } \\
\text { euskera }\end{array}$} & \multirow{2}{*}{ Con -a léxica } & $\begin{array}{l}\text { Euskera: Gazta hori / } \\
\text { txuri } \\
\text { Castellano: Queso } \\
\text { amarillo / blanco }\end{array}$ & $\begin{array}{l}\text { Euskera: Kandela gorri / txur } \\
\text { Castellano: Vela roja / blanca }\end{array}$ \\
\hline & & $\begin{array}{l}\text { Euskera: labana txuri / } \\
\text { beltz } \\
\text { Castellano: cuchillo blanco } \\
\text { / negro }\end{array}$ & $\begin{array}{l}\text { Euskera: tipula txuri / gorri } \\
\text { Castellano: cebolla blanca / } \\
\text { roja }\end{array}$ \\
\hline & \multirow{2}{*}{ Sin-a léxica } & $\begin{array}{l}\text { Euskera: arrain hori / } \\
\text { beltz } \\
\text { Castellano: pez amarillo } \\
\text { / negro }\end{array}$ & $\begin{array}{l}\text { Euskera: gezi gorri / beltz } \\
\text { Castellano: flecha roja / negra }\end{array}$ \\
\hline & & $\begin{array}{l}\text { Euskera: arkatz luze / } \\
\text { motz } \\
\text { Castellano: lapiz largo / } \\
\text { corto }\end{array}$ & $\begin{array}{l}\text { Euskera: sagar gorri / hori } \\
\text { Castellano: manzana roja / } \\
\text { amarilla }\end{array}$ \\
\hline
\end{tabular}

Tabla 3. Características de los SSNN correspondientes a los objetos utilizados en la tarea de elicitación. (Adaptada de Parafita Couto et al. 2014).

Dicha tarea permitió obtener 58 ejemplos de cambios de código en las 15 parejas analizadas, aunque la mayoría de ellos eran cambios de lengua entre cláusulas, o sintagmas determinante denominados "islas". Solo se obtuvo un ejemplo (6) cuyo sustantivo estuviese en castellano y el determinante en euskera ${ }^{6}$. En este ejemplo (6) el género femenino del determinante puede explicarse tanto mediante el género analógico (cebolla es femenino) como por la forma fonológica del sustantivo (terminación en $-a$ ).

6.- En este estudio también se analizó la posible correspondencia entre las actitudes de los hablantes hacia el cambio de código y el número de enunciados mixtos y sintagmas nominales mixtos, sin embargo, no se obtuvo ninguna correlación estadísticamente significativa, debido probablemente al escaso número de ejemplos observados. 
(6) Gero gezi-a beltz-a, baina begiratzen o sea hacia la... hacia [la tipula], ulertzen? Luego flecha-D negra-D pero mirando o sea hacia la... hacia la cebolla, entiendes Luego la flecha negra, pero mirando o sea hacia la... hacia la cebolla, ¿entiendes?

\subsubsection{Tarea de juicios de aceptabilidad de estímulos auditivos}

En la segunda tarea experimental se recogieron los juicios de aceptabilidad de los mismos hablantes que participaron en la tarea de elicitación, pero esta vez sobre una serie de estímulos auditivos con enunciados mixtos. Se presentaron 88 enunciados mixtos de los cuales 64 eran experimentales. En las oraciones experimentales se mantuvo constante el marco oracional en castellano y solo variaron los elementos del sintagma determinante (7) (ver sección 2.2.1). Se controló la terminación fonológica de los sustantivos en euskera así como el género de los equivalentes en castellano y se utilizaron elementos léxicos que habían sido producidos en SSNN mixtos en el corpus (Deuchar et al. 2010). También se controlaron otras variables, tales como la lengua de los adjetivos, etc. (véase Parafita Couto et al. 2014 para más detalles).

$$
\begin{aligned}
& \text { El hombre ha comprado [la tipula txuri] } \\
& \text { El hombre ha comprado [D.fem cebolla blanca] } \\
& \text { El hombre ha comprado la cebolla blanca }
\end{aligned}
$$

Se realizaron dos tipos de análisis ${ }^{7}$. En el primer análisis se seleccionaron las respuestas más rápidas (menos de 4000 ms) con mayor grado de convergencia entre los participantes y se hizo un análisis descriptivo de las 24 oraciones experimentales a fin de obtener una impresión general de las preferencias de los participantes. La mayoría (71\%) de las respuestas más automáticas eran rechazadas por más del $65 \%$ de los participantes. Además, entre las respuestas rechazadas, prevalecían las que tenían adjetivo en castellano (dos cambios de código) y cuyo género era incongruente con el género del sustantivo (8). Entre los estímulos aceptados, la mayoría contenían un adjetivo en euskera y el determinante castellano femenino, si bien, en su mayoría los sustantivos de estos estímulos contenían una terminación acorde con el género del equivalente castellano, congruente con el femenino (9). Así pues, las respuestas más rápidas no permitieron analizar en detalle los posibles efectos de la forma fonológica y el género fonológico para la asignación del género.

(8) El hombre ha comprado [la tipul blanco] El hombre ha comprado [D.fem cebolla blanco]

El hombre ha comprado la cebolla blanco

7.- Al igual que en la tarea de juicios y a diferencia de lo observado por Parafita Couto et al. (2015) en el estudio del galés-inglés, no se encontró ninguna correlación estadísticamente significativa entre las actitudes de los hablantes y el grado de aceptabilidad en la tarea de los juicios. 
(9) El hombre ha comprado [la sagarr-a gorri-a]

El hombre ha comprado [D.fem manzana-D roja-D]

El hombre ha comprado la manzana roja

En el segundo análisis se comparó el nivel de aceptabilidad según las variables principales del estudio: terminación fonológica y género del equivalente. A pesar de que ambas variables parecen influir en el grado de aceptabilidad de los participantes, la forma fonológica ejerce una mayor influencia en la asignación del género en las construcciones nominales. Tal y como puede verse en la figura 4 , los determinantes femeninos se prefieren en todas las condiciones, excepto en aquellas en las que el género del determinante es congruente con el del nombre pero la terminación fonológica es incongruente, ya que en estos casos el determinante masculino (10) y el femenino (11) se aceptan de una manera similar.

(10) El hombre ha comprado [el ilarr-a lodi]

El hombre ha comprado [D.masc guisante-D gordo]

El hombre ha comprado el guisante gordo

(11) El hombre ha comprado [la tipul txuri]

El hombre ha comprado [D.fem cebolla blanca]

El hombre ha comprado la cebolla blanca

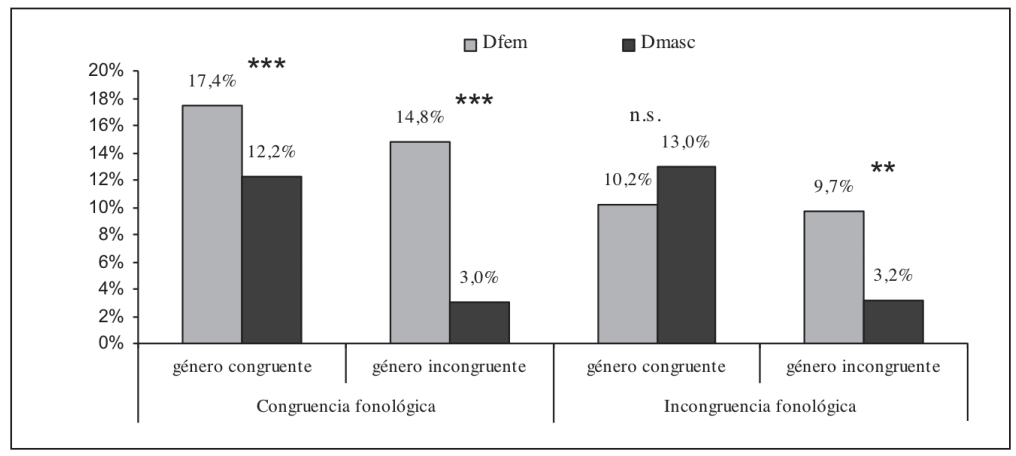

Figura 4. Grado de aceptabilidad para las distintas condiciones de congruencia e incongruencia de género y de terminación fonológica ${ }^{8}$. (Adaptada de Parafita Couto et al. 2014).

Además, se observó que cuando ambas variables son incongruentes con el género del determinante, había una preferencia por SSNN con determinante femenino (12) frente a

8.- Siguiendo las convenciones internacionales se han utilizado los siguientes símbolos: ${ }^{* *} p<0,01 ;{ }^{* * *} p<$ 0,$001 ;$ n.s. $=$ no significativo . 
los que tenían masculino (13) (Figura 4). Es decir, los juicios de aceptabilidad confirmaron la asimetría observada en los datos naturales según la cual los determinantes femeninos se aceptan con sustantivos cuyo equivalente es femenino $(8,9)$ y también cuando el equivalente es masculino (12), pero sin embargo los determinantes masculinos solo se aceptan de manera similar al determinante femenino con sustantivos cuyo equivalente es masculino (10). Esta preferencia general por el determinante femenino incluso cuando el género y la terminación fonológica son incongruentes, parece indicar que el género femenino puede ser el género por defecto en los SSNN mixtos euskera-castellano.
El hombre ha comprado [la ilar lodi]
El hombre ha comprado [D.fem guisante gordo]
El hombre ha comprado la guisante gordo
El hombre ha comprado [el
tipula txuri]
El hombre ha comprado [D.masc cebolla blanca]
El hombre ha comprado el cebolla blanca

En resumen, en Parafita Couto et al. (2014) se han analizado construcciones nominales castellano-euskera para ver los factores que guían la asignación del género mediante diferentes métodos. El análisis del corpus indica que puede haber una preferencia por el determinante femenino, pues este aparece junto con sustantivos cuyo equivalente es masculino y femenino. La tarea de elicitación no ha resultado valiosa debido a la escasez de ejemplos de la estructura de interés, fenómeno que puede deberse a la libertad de la tarea. Sin embargo, los resultados de los juicios de aceptabilidad han permitido ver que los resultados del corpus natural y de los juicios convergen en indicar que el femenino actúa como género por defecto en las construcciones nominales mixtas castellano-euskera debido a la posible reinterpretación del determinante del euskera ( $-a)$ como marca de género femenino. Aparentente, estos resultados difieren de los observados en las construcciones nominales mixtas castellano-inglés donde se observa que el género por defecto es el masculino (Dussias et al. 2013; Jake et al. 2002; Poplack, Pousada \& Sankoff 1982). Todo ello parece indicar que los recursos que los bilingües usan para la asignación del género pueden variar con respecto a los de los monolingües. A pesar de la convergencia de los datos naturales y experimentales, sería preferible completar el estudio con datos de juicios inconscientes obtenidos mediante tareas electrofisiológicas. Asimismo, en vista de que la tendencia hacia el femenino se observa en hablantes L1 (corpus) y L2 de euskera (juicios), sería interesante ver si el patrón se mantiene en cada una de las tareas dependiendo del tipo de bilingües estudiado (cf. Liceras et al. 2008).

\subsection{Papiamento-holandés}

En el habla bilingüe papiamento-holandés la construcción nominal es también un potencial "punto de conflicto" cuando hay un adjetivo de una lengua y un sustantivo de la otra, ya que el papiamento, al igual que el español, el euskera, o el galés, tiene adjetivos postnominales (14) mientras que el holandés, al igual que el inglés, tiene adjetivos prenominales (15). Además, el holandés tiene género gramatical (género no común y género común), mientras que el papiamento no tiene género gramatical. 
biña còrá [Papiamento]

vino tinto

rode wijn [Holandés]

tinto vino

\subsubsection{Datos de corpus}

Teniendo esto en cuenta, Parafita Couto y Gullberg (en preparación) obtuvieron 77 construcciones nominales mixtas del corpus bilingüe papiamento-holandés del Max Planck Institute for Psycholinguistics en Nijmegen (Holanda). Los resultados indican que los cambios de código entre el determinante y el sustantivo son los más comunes en este corpus, con la combinación determinante del papiamento y sustantivo del holandés como la más numerosa. Esto iría en contra de las predicciones generativistas de que la lengua que provee el determinante es la lengua con más rasgos phi (cf. Liceras et al. 2008), ya que el papiamento no tiene el rasgo phi de género mientras que el holandés sí lo tiene. En cuanto a los adjetivos, los adjetivos antepuestos son los más comunes, resultado que llama la atención ya que en papiamento se prefieren adjetivos postnominales. Parece que en este caso, la predicción generativista se aproxima más a una explicación de los datos, pero el resultado del corpus en esta comunidad va en contra de lo que se encontró para la población bilingüe de Gales. Además, los resultados de la lengua del determinante y los resultados de la posición del adjetivo apuntan en direcciones contrarias: los primeros no apoyan las predicciones generativistas mientras que los segundos sí lo hacen.

\subsubsection{Tarea electrofisiológica}

Para ver si los hallazgos de Parafita Couto et al. (en revisión) sobre el galés-inglés son generalizables a otros pares de lenguas, Parafita Couto y colaboradores (Schiller, Pablos, Sattaur \& Parafita Couto 2013; Schiller, Pablos \& Parafita Couto, 2014) replicaron el mismo estudio con hablantes bilingües papiamento-holandés. Usaron el mismo diseño experimental mediante el método de potenciales evocados y testaron 20 bilingües papiamento-holandés. Al igual que en el estudio del galés-inglés, manipularon (1) la lengua matriz, que los autores identificaron con la del verbo de la oración, (2) la lengua del adjetivo, (3) la lengua del sustantivo, y (4) el orden de las palabras.

Al contrario que en el estudio del galés-inglés, se encontró un N400 en la condición no prevista entre las predicciones generativistas. En cambio, la lengua matriz puede dar cuenta de los diferentes resultados: una lengua matriz que es familiar para los participantes resulta mejor que una lengua matriz que no es tan familiar para ellos (es decir, la lengua matriz inglés sería la menos común para los bilingües inglés-galés y la lengua matriz holandés sería la menos común para los bilingües papiamento-holandés).

Parafita et al. (en preparación) llegaron a la conclusión de que hacer caso omiso de la realidad sociolingüística puede llevar a interpretaciones erróneas. Se debe siempre apoyar en las observaciones de los corpus multilingües a la hora de interpretar los datos experimentales. Teniendo esto en cuenta, se están llevando a cabo nuevos experimentos en este momento para resolver el debate teórico. 


\section{Conclusiones}

El objetivo de este artículo es ofrecer un panorama general sobre las metodologías utilizadas para estudiar las características gramaticales del cambio de código y abordar una de las cuestiones metodológicas fundamentales, es decir, cuál es o cuáles son los métodos más adecuados para estudiar los aspectos gramaticales del cambio de código sin comprometer dicho fenómeno (cf. Gullberg et al. 2009). En primer lugar, se han revisado los aspectos positivos y las limitaciones de algunas de las metodologías, y después se han presentado datos empíricos de estudios realizados en tres comunidades bilingües (galés-inglés, euskera-castellano y papiamento-holandés) en los que se han recolectado datos mediante dichas metodologías para explorar algunos de los "puntos de conflicto" que surgen a nivel del sintagma nominal.

En general, los estudios de las tres comunidades bilingües corroboran la conveniencia de integrar distintas metodologías (tareas más próximas a los datos naturales y tareas experimentales más restrictivas) para poder analizar en profundidad tanto las propiedades lingüísticas como las extralingüísticas del cambio de código (González-Vilbazo et al. 2009; Gullberg et al. 2009; MacSwan y MacAlister 2010).

El estudio del galés-inglés en el que se han combinado todas las metodologías abordadas en este artículo ha permitido observar que a pesar de que los datos naturales pueden resultar informativos y sugerentes para el estudio de los aspectos gramaticales de cambio de código, parece más apropiado y efectivo recoger datos de producción de tareas de elicitación semiespontánea, ya que con un coste muchísimo menor se ha conseguido obtener una mayor cantidad de las construcciones nominales mixtas deseadas. Sin embargo, los datos de producción no resultan concluyentes para responder a cuestiones teóricas, ya que no siempre se encuentran (suficientes) ejemplos de las distintas combinaciones (lógicamente) posibles.

Se ha visto que es necesario recurrir a los juicios de los hablantes mediante tareas experimentales que permiten obtener intuiciones sobre estructuras con mayor variedad de condiciones. Pero como hemos comprobado, en algunas comunidades, como es la del galésinglés, los juicios de gramaticalidad se pueden ver condicionados por las actitudes de los hablantes hacia el cambio de código. Es por eso que, en caso de disponer de los medios para ello, las tareas electrofisiológicas son una buena alternativa, ya que nos permiten acceder a la reacción inconsciente del hablante. Somos conscientes de que no siempre es posible tener a mano la tecnología y los medios necesarios para poder realizar investigaciones tan exhaustivas sobre el fenómeno.

Una de las principales implicaciones de este trabajo es la convergencia de los resultados de los diferentes métodos observada en los estudios del galés-inglés y del euskera-castellano. Los patrones observados en tareas más naturales se han corroborado mediante tareas más restrictivas, es decir, se ha observado que en general convergen los resultados de tareas noexperimentales y experimentales (juicios y/o potenciales evocados). Las tareas experimentales, además, aportan datos que no se pueden obtener mediante tareas no-experimentales, puesto que proporcionan evidencia negativa, es decir, permiten conocer qué estructuras rechazan los hablantes. 
A pesar de que ha habido amplia investigación sobre el cambio de código desde un punto de vista sociolingüístico y estructural (principalmente mediante el uso de corpus y datos de juicios de aceptabilidad), solo estamos empezando a comprender los mecanismos neurocognitivos que subyacen a este tipo de comportamiento lingüístico. Es necesario poder traducir las predicciones que las teorías lingüísticas hacen sobre el cambio de código intraoracional a hipótesis que puedan ser comprobadas experimentalmente. Por otro lado, es muy importante no olvidar que las propiedades extralingüísticas de la comunidad estudiada pueden ser cruciales a la hora de interpretar los datos, tal y como hemos visto en los diferentes resultados obtenidos en cuanto al grado de aceptabilidad en los juicios del galés-inglés y euskera-castellano, así como en los resultados preliminares obtenidos en los estudios de ERPs con galés-inglés y papiamento-holandés. Finalmente, es importante estudiar distintos tipos de bilingües en cuanto a dominio, uso y edad de adquisición para ver si los patrones de cambio de código están determinados por las propiedades estructurales de las lenguas per se o se ven afectados por otro tipo de factores extralingüísticos. 


\section{Referencias}

Bentahila, Abdelali \& Eirlys E. Davies. 1983. The syntax of Arabic-French code-switching. Lingua 59, 301-330.

Berk-Selingson, Susan. 1986. Linguistic constraints on intrasentential code-switching: A study of Spanish/Hebrew bilingualism. Language in Society 15, 313-348.

Cantone, Katja Francesca \& Jeff MacSwan. 2009. Adjectives and word order: A focus on Italian-German codeswitching. En Kees de Bot, Donald Winford \& Ludmila Isurin (eds.), Multidisciplinary approaches to codeswitching, 243-278. Amsterdam: John Benjamins Publishing.

Chan, Brian Hok-Shing. 2008. Code-switching, word order and the lexical/functional category distinction. Lingua 118, 777-809.

De Bot, Kees. 1992. A bilingual production model: Levelt’s 'Speaking' model adapted. Applied Linguistics 13, 1-24.

De Bot, Kees. 2003. Bilingual speech: from concepts to articulation. Fremdsprachen Lehren Und Lernen 32, 91-103.

Deuchar, Margaret, Peredur Davies, Jon Herring, Mari Carmen Parafita Couto \& Diana Carter. 2014. Building bilingual corpora. En Enlli Môn Thomas \& Ineke Mennen (eds.), Advances in the Study of Bilingualism, 98-110. Bristol: Multilingual Matters.

Deuchar, Margaret, Irantzu Epelde, Bernard Oyharçabal \& Mari Carmen Parafita Couto. 2010. Gender agreement in Spanish-Basque and Spanish-English nominal constructions. Comunicación presentada en el XXXIX Simposio Internacional de la SEL, Santiago de Compostela, España.

Deuchar, Margaret. 2012. Code switching. En Carol A. Chapelle (ed.), Encyclopedia of Applied Linguistics, 657-664. New York: Wiley.

Dussias, Paola E., Jorge R. Valdés Kroff, Rosa E. Guzzardo Tamargo \& Chip Gerfen. 2013. When gender and looking go hand in hand: Grammatical gender processing in L2 Spanish. Studies in Second Language Acquisition 35, 353-587.

Epelde, Irantzu \& Bernard Oyharçabal. 2010. Perpaus barneko hizkuntza lerratzeak ekialdeko euskal elebidunetan. En Beatriz Fernández, Pablo Albizu \& Ricardo Etxepare (eds.), Euskara eta euskarak: aldakortasun sintaktikoa aztergai, 55-65. Bilbao: Universidad del País Vasco.

Ezeizabarrena, Maria José \& Amaia Munarriz. 2012. Orden de palabras en los enunciados mixtos (euskera-castellano) de bilingües típicos y atípicos. En Ana María Cestero Mancera, 
Isabel Molina Martos \& Florentino Paredes García (eds.), La lengua, lugar de encuentro. Actas del XVI Congreso Internacional de la ALFAL (Alcalá de Henares, 6-9 de junio de 2011), 3893-3902. Alcalá de Henares: Servicio de Publicaciones de la Universidad de Alcalá.

Ezeizabarrena, Maria-José. 2009. Development in language mixing: early Basque-Spanish bilingualism. En John Grinstead (ed.), Hispanic child languages: typical and impaired development, 57-89. Amsterdam: John Benjamins.

Featherston, Samuel. 2007a. Data in generative grammar: The stick and the carrot. Theoretical Linguistics 33, 269-318

Featherston, Samuel. 2007b. Reply. Theoretical Linguistics 33, 401-413.

Featherston, Samuel. 2008. Thermometer judgements as linguistic evidence. En Claudia Maria Riehl \& Astrid Rothe (eds.), Was ist linguistische Evidenz?. Aachen: Shaker Verlag.

Featherston, Samuel. 2009. Relax, lean back, and be a linguist. Zeitschrift für Sprachwissenschaft 28, 127-132.

González-Vilbazo, Kay, Laura Bartlett, Sarah Downey, Shane Ebert, Jeanne Heil, Bradely Hoot, Bryan Koronkiewicz \& Sergio Ramos. 2013. Methodological considerations in codeswitching research. Studies in Hispanic and Lusophone Linguistics 6, 119-138.

Gullberg, Marianne, Peter Indefrey \& Pieter Muysken. 2009. Research techniques for the study of code-switching. En Barbara E. Bullock \& Almeida Jacqueline Toribio (eds.), The Cambridge Handbook on linguistic code-switching, 21-39. Cambridge: Cambridge University Press.

Harris, James. 1991. The exponence of gender in Spanish. Linguistic Inquiry 22, 27-62.

Herring, Jon, Margaret Deuchar, Mari Carmen Parafita Couto \& Mónica Moro Quintanilla. 2010. I saw the madre: Evaluating predictions about codeswitched determiner noun sequences using Spanish-English and Welsh-English data. International Journal of Bilingual Education and Bilingualism 13, 553-573.

Ibarra, Orreaga. 2011a. Nafar euskaldun gazteen ahozko diskurtsoa. Iruñea: Nafarroako Unibertsitatea Publikoa.

Ibarra, Orreaga. 2011b. Sobre estrategias discursivas de los jóvenes vascohablantes: causas que motivan el cambio de código. Oihenart 26, 277-298.

Jake, Janice L., Carol Myers-Scotton \& Steven Gross. 2005. A response to MacSwan (2005): Keeping the Matrix Language. Bilingualism: Language and Cognition 8, 1-6.

Jake, Janice L., Carol Myers-Scotton \& Steven Gross. 2002. Making a minimalist approach to 
codeswitching work: Adding the Matrix Language. Bilingualism: Language and Cognition 5, 69-91.

Koronkiewicz, Bryan \& Shane Ebert. 2014. Methods in code-switching research: When does modality matter? Comunicación presentada en UIC BilForum, University of Illinois Chicago.

Kroll, Judith F. \& Natasha Tokowicz. 2005. Models of bilingual representation and processing. En Judith F. Kroll \& Annette M. B. de Groot (eds.), Handbook of bilingualism: psycholinguistic approaches, 531-553. New York: Oxford University Press.

Kutas, Marta, Eva M. Moreno \& Nicole Y. Y. Wicha. 2009. Code-switching and the brain. En B. E. Bullock \& A. J. Toribio (eds.), The Cambridge Handbook of linguistic code-switching, 289-306. Cambridge: Cambridge University Press.

Labov, William. 1975. Empirical foundations of linguistic theory. In Robert Austerlitz (ed.), The Scope of American Linguistics, 77-133. Lisse: The Peter de Ridder Press.

Lantto, Hanna. 2014. Code-switching, swearing and slang: The colloquial register of Basque in Greater Bilbao. International Journal of Bilingualism 18, 633-648.

Liceras, Juana M., Raquel Fernández Fuentes, Susana Perales, Rocío Pérez-Tattam \& K. Todd Spradlin. 2008. Gender and number agreement in bilingual native and non-native grammars: a view from child and adult functional-lexical mixings. Lingua 118, 761-852.

MacSwan, Jeff \& Kara T. McAlister. 2010. Naturalistic and elicited data in grammatical studies of codeswitching. Studies in Hispanic and Lusophone Linguistics 3, 521-532.

MacSwan, Jeff. 1999. A minimalist approach to intrasentential code switching. New York: Garland Publishing.

MacSwan, Jeff. 2005. Codeswitching and generative grammar: A critique of the MLF model and some remarks on "modified minimalism". Bilingualism: Language and Congition 8, 1-22.

MacSwan, Jeff. 2009. Generative approaches to code-switching. En B. E. Bullock \& A. J. Toribio (eds.), The Cambridge handbook of linguistic code-switching 309-335. New York: Cambridge University Press.

Moyer, Melissa G. 1992. Analysis of code-switching in Gibraltar. Tesis doctoral, Universitat Autònoma de Barcelona.

Myers-Scotton, Carol. 1992. Constructing the frame in intrasentential codeswitching. Multilingua 11, 101-128.

Myers-Scotton, Carol. 2002. Contact Linguistics. Oxford: Oxford University Press. 
Oyharçabal, Bernard, Jasone Salaberria \& Irantzu Epelde. 2013. Kode aldaketa euskara-frantsesa eta euskara-gaztelania elebidunetan: ACOBA egitasmoa / El cambio de código en hablantes bilingües euskera-francés y euskera-español: el proyecto ACOBA. Comunicación presentada en "Bilingüismo y contacto lingüístico en el País Vasco y en otras comunidades / Elebitasuna eta hizkuntza kontaktua Euskal Herrian eta beste Erkidego batzuetan", Donostia.

Oyharçabal, Bernard, Jasone Salaberria \& Irantzu Epelde. 2015. ACOBA: Bilingual speech: code-switching among Spanish-Basque and French-Basque speakers. Base de datos disponible en la siguiente página web: http://acobaiker.org.

Parafita Couto, M. Carmen \& Marianne Gullberg. En preparación. Code-switching within the noun phrase - Evidence from three corpora.

Parafita Couto, M. Carmen, Amaia Munarriz, Irantzu Epelde, Margaret Deuchar \& Bernard Oyharçabal. 2014. Gender conflict resolution in Spanish-Basque mixed DPs. Bilingualism, Language and Cognition. DOI: 10.1017/S136672891400011X.

Parafita Couto, M. Carmen, Bastien Boutonnet, Noriko Hoshino, Peredur Davies, Margaret Deuchar \& Guillaume Thierry. En revisión. Testing alternative theoretical accounts of code-switching using event-related brain potentials.

Parafita Couto, Mari Carmen, Margaret Deuchar \& Marika Fusser. 2015. How do WelshEnglish bilinguals deal with conflict? Adjective-noun order resolution. En Gerald Stell \& Kofi Yakpo (eds.), Code-switching at the crossroads between structural and sociolinguistic perspectives. Mouton de Gruyter.

Poplack, Shana, Alicia Pousada \& David Sankoff. 1982. Competing influences on gender assignment: variable process, stable outcome. Lingua 57, 1-28.

Poplack, Shana. 1980. Sometimes I'll start a sentence in Spanish y termino en español: toward a typology of code-switching. Linguistics 18, 581-618.

Roca, Ignacio M. 1989. The organisation of grammatical gender. Transactions of the Philological Society $87,1-32$.

Roca, Ignacio M. 2005. La gramática y la biología en el género del español. Revista Española de Lingüística 35, 17-44.

Schiller, Niels O., Leticia Pablos \& Mari Carmen Parafita Couto. 2014. Resolving PapiamentoDutch Conflicts. Comunicación presentada en "Cognitive Neuroscience Society", Boston.

Schiller, Niels O., Leticia Pablos, Raiza Sattaur \& Mari Carmen Parafita Couto. 2013. No trespassing! Papiamentu-Dutch conflict sites. Comunicación presentada en "Society for the Neurobiology of Language", San Diego. 
Schütze, Carson T. 1996. The empirical base of linguistics: Grammaticality judgments and linguistics methodology. Chicago: The University of Chicago.

Tremblay, Annie. 2005. Theoretical and methodological perspectives on the use of grammaticality judgment tasks in linguistic theory. Second Language Studies 24, 129-167.

Van Hell, Janet G, Kaitlyn A. Litcofsky \& Caitlin Y. Ting. en prensa, 2015. Sentential codeswitching: cognitive and neural approaches. En John W. Schwieter (ed.), The Cambridge Handbook of Bilingual Processing.

Weinreich, Uriel. 1953. Languages in contact, Findings and problems. New York: Linguistic Circle of New York. 